\title{
A GENERALIZED JACOBIAN CRITERION FOR REGULARITY
}

\author{
PETER SEIBT
}

\begin{abstract}
For a commutative noetherian algebra $B$ over a perfect field $A$ regularity is equivalent to the flatness of $\Omega_{B \mid A}$ plus $H_{1}(A, B, B)=0$ (simplicial homology). In characteristic 0 the homological condition is superfluous.
\end{abstract}

1. Introduction. All rings to be considered will be commutative and unitary. The classical Jacobian criterion (in terms of Kähler differentials) reads like this: Let $A$ be a perfect field, $(B, \mathrm{~m}, K)$ a local $A$-algebra of finitely generated type (obtained by localizing an $A$-algebra of finite type) such that $K=B / \mathfrak{m}=A$ and let $\Omega_{B \mid A}$ be the $B$-module of $A$-differentials for $B$. Then $B$ is regular if and only if $\Omega_{B \mid A}$ is free (of rank' equal to the dimension of $B$ ).

I want to generalize this differential characterization of regularity in the following way.

THEOREM. Let $A$ be a perfect field, $B$ a noetherian $A$-algebra. Then $B$ is regular (all localizations $B_{\mathfrak{m}}$ of $B$ at maximal ideals $m$ are regular local rings) if and only if the following two conditions hold:

(1) $\Omega_{B \mid A}$ is B-flat.

(2) For every infinitesimal A-extension

$$
E=0 \rightarrow M \rightarrow C \rightarrow B \rightarrow 0 \text { of } B,
$$

the associated derived module sequence

$$
\operatorname{diff}(E)=0 \rightarrow M \rightarrow \Omega_{C \mid A} \otimes_{C} B \rightarrow \Omega_{B \mid A} \rightarrow 0
$$

is exact.

Corollary. If $\operatorname{char} A=0$, we have

$B$ is regular if and only if $\Omega_{B \mid A}$ is $B$-flat.

I do not know whether condition (2) is really relevant in positive characteristic ( $A$ perfect, of course).

2. Proof of the theorem. Recall for arbitrary $A \rightarrow B$ the two sequences $\left(H_{n}(A, B,-)\right)_{n \geqslant 0}$ and $\left(H^{n}(A, B,-)\right)_{n \geqslant 0}$ of simplicial homology and cohomology functors as defined and discussed in [1].

Received by the editors February 13, 1984.

1980 Mathematics Subject Classification. Primary 13D03, $13 \mathrm{H} 05$.

Key words and phrases. Regular local ring, differentials, simplicial (co)homology. 
LEMMA. For arbitrary $A \rightarrow B$ the following three conditions are equivalent:

(a) $\operatorname{Ext}_{B}^{1}\left(\Omega_{B \mid A},-\right)=H^{1}(A, B,-)$.

(b) $H_{1}(A, B, B)=0$.

(c) $H_{1}(A, B,-)=\operatorname{Tor}_{1}^{B}\left(\Omega_{B \mid A},-\right)$.

Condition (a) needs some comment:

For every $B$-module $M$, $\operatorname{Ext}_{B}^{1}\left(\Omega_{B \mid A}, M\right)$ classifies the singular 1-extensions of $\Omega_{B \mid A}$ by $M$, whereas $H^{1}(A, B, M)$ classifies the infinitesimal $A$-extensions of $B$ by $M$. There is a $B$-monomorphism $\operatorname{Ext}_{B}^{1}\left(\Omega_{B \mid A}, M\right) \rightarrow H^{1}(A, B, M)$, functorial in $M$, whose image consists of the classes of those $A$-extensions $E=0 \rightarrow M \rightarrow C \rightarrow B \rightarrow 0$, which have an exact derived module sequence

$$
\operatorname{diff}(E)=0 \rightarrow M \rightarrow \Omega_{C \mid A} \otimes_{C} B \rightarrow \Omega_{B \mid A} \rightarrow 0
$$

(see [3, pp. 158-161]). Thus (a) is only another formulation of condition (2) of the theorem.

Proof of The Lemma. (a) implies (b). We have $H^{1}(A, B, W)=0$ for every injective $B$-module $W$. But for injective $B$-modules

$$
H^{1}(A, B, W)=\operatorname{Hom}_{B}\left(H_{1}(A, B, B), W\right)
$$

by $\left[1,3.21\right.$, p. 42]. Now take $W$ the injective hull of $H_{1}(A, B, B)$.

(b) implies (c). This follows from [1, 3.19, p. 41].

(c) implies (a). We have immediately $H_{1}(A, B, B)=0$, and thus $H^{1}(A, B, W)=0$ whenever $W$ is $B$-injective (see (a) $\Rightarrow(\mathrm{b})$ ). But by [2], $H^{1}(A, B,-)$ is part of an exact connected sequence of cohomology functors, say $\left(D^{n}(B \mid A,-)\right)_{n \geqslant 0}$, where $D^{0}(B \mid A,-)=\operatorname{Hom}_{B}\left(\Omega_{B \mid A},-\right), D^{1}(B \mid A,-)=H^{1}(A, B,-)$ and $D^{n}(B \mid A,-)$ $=\operatorname{Ext}_{B}^{n-1}(K,-), n \geqslant 2$, for some $B$-module $K$. The vanishing on injective coefficients in positive degree yields, in particular, $H^{1}(A, B,-)=\operatorname{Ext}_{B}^{1}\left(\Omega_{B \mid A},-\right)$.

This finishes the proof of the lemma.

Now the theorem follows easily:

By the lemma (condition (b)) we may assume $B$ to be local. Since $A$ is perfect, the regularity of $B$ is equivalent to the formal smoothness of $B$ over $A$. But by [1, Theorem 30, p. 331] this in turn is equivalent to $H_{1}(A, B,-)=0$.

The lemma now immediately yields the assertion of the theorem.

As to the corollary, we may rest in the local case. The flatness of $\Omega_{B \mid A}$ gives the $B \mid \mathrm{m}^{k}$-projectivity of $\Omega_{B \mid A} \otimes_{B} B / \mathrm{m}^{k}$ for every $k \geqslant 1\left(\mathrm{~m} / \mathrm{m}^{k}\right.$ is nilpotent, $\mathrm{m}$ the maximal ideal of $B$, of course).

A theorem of Radu's (cf. [1, 7.31, p. 103]) guarantees the regularity of $B$.

\section{REFERENCES}

1. M. André, Homologie des algèbres commutatives, Springer-Verlag, Berlin, Heidelberg and New York, 1974.

2. M. Barr, A cohomology theory for commutative algebras. I, II, Proc. Amer. Math. Soc. 16 (1965), 1379-1391.

3. L. Illusie, Complexe cotangent et déformations. I, Lecture Notes in Math., vol. 239, Springer-Verlag, Berlin, Heidelberg and New York, 1971.

U. E. R. De Mathematiques et d'Informatique, Universite de Bordeaux I, F - 33405 Talence, France 